\title{
DOS EJEMPLOS DE SOLUCIÓN DE PROBLEMAS DE CONSERVACIÓN EN PIEZAS CERÁMICAS DEL MUSEO PROVINCIAL DE MURCIA
}

\author{
A. MADROÑERO DE LA CAL
}

\begin{abstract}
Se estudia un proceso de alteración química de materiales arqueológicos en los museos, concretamente de unos azulejos vidriados de época moderna y de platos con decoración de cobre, proponiéndose las soluciones más adecuadas para su conservación.
\end{abstract}

The process of chemical alterations of archaeological materials in the Museums is studied, particulary the process of damage of modern glazed tiles and of the plates with copper decoration. Appropiate methods of treatement are proposed.

\section{INTRODUCCIÓN}

Todos tenemos un amplio conocimiento acerca de los problemas de conservación que presentan las piezas metálicas expuestas en nuestros museos. Pero las piezas cerámicas suelen resisitir, por su propia naturaleza, el paso del tiempo y de ambientes tan acondicionados como es el interior de un museo, que tenemos la sensación de que, en este campo, la problemática no existe.

Quizá por ello pueda ser de algún interés un par de casos reales, relativos a piezas existentes en el $\mathrm{Mu}$ seo Provincial de Murcia, y que pueden servir de ilustración de cómo una bienintencionada solución, inspirada solamente en el sentido común, puede producir un daño irreparable. Por el contrario, si se actúa analizando el mecanismo de dañado de las piezas, existe siempre una solución razonable.

\section{EL PROBLEMA \\ DEL RESQUEBRAJAMIENTO DEL VIDRIADO DE AZULEJOS}

Existen en el Museo Provincial de Murcia unos azulejos de los siglos XVIII y XIX que presentan el problema de que su vidriado se resquebraja y ahueca sin que nadie les toque en las vitrinas. Interesaría conocer el proceso mediante el cual se produce este deterioro a fin de detenerlo en los todavía no dañados, así como para sanear los ya dañados, antes de proceder a su restauración.

El único defecto durante la conservación al que puede culparse, a falta de otro, de este deterioro es la falta de estanqueidad de las vitrinas, lo que conlleva a que la humedad ambiental pueda actuar sobre las piezas. La humedad ambiental, aunque no ha sido medida correctamente, es evidentemente elevada, ya que los saquitos de gel de sílice que se sitúan en el interior de las vitrinas para desecarlas tienen una vida útil muy corta.

El problema básico es explicar cómo una pieza como es un azulejo (un metal o una madera sería completamente diferente) puede verse afectado hasta tal grado por algo tan aparentemente inocuo como es la humedad. Pensemos, por ejemplo, en vasijas que, sin la protección del vidriado y con una composición similar a la de la galleta, llevan conteniendo agua durante siglos sin alterarse. De hecho, el mecanismo de absorción de la humedad de los ladrillos empleados en la edificación y su desmoronamiento a causa de las heladas (dilatación del agua 
al producirse las heladas nocturnas) resulta impensable en nuestro caso, ya que nunca se alcanzan en el interior del Museo las temperaturas bajo cero que este proceso requerían.

Nuestro único punto de partida son estos dos hechos:

a) El único agente posible es la humedad. Otros factores como cambios bruscos de temperatura, vibraciones mecánicas o golpes, etc., han de ser desechados de entrada.

b) El proceso ha tardado mucho en actuar, pues han estado los azulejos durante decenios sin haber presentado deterioro alguno.

El examen visual de los azulejos nos permite establecer las observaciones siguientes, que esquematizamos en fig. 1. El vidriado presenta una fractura frágil sin que pueda advertirse dónde se inició la rotura (como seguro que se podría observar en el caso de que el dañado hubiese sido originado, por ejemplo, con el golpe de una herramienta punzante) y lo que llama poderosamente la atención es un depósito de una sustancia blancuzca pulverulenta y esponjosa que parece haberse depositado entre el vidriado y la galleta. Lo más curioso es que por la simple apariencia, cabe pensar que esta sustancia, además de propiciar el despegado del vidriado, indujo especialmente en los puntos alejados de los bordes, un resquebrajado del vidriado. En las zonas próximas a los bordes el desplegado es algo menor, menos extensivo que en el centro del azulejo; ello nos hace pensar que la proximidad al borde del azulejo tiene alguna influencia en el proceso.

En esta sustancia blancuzca debe, lógicamente, estar la clave del proceso destructivo. Llama mucho la atención el que se haya formado en un lugar tan inaccesible y alejado de la atmósfera, y el que no se forme en, por ejemplo, los azulejos adheridos a una pared. El hecho de que además de propiciar el despegado originase la rotura del vidriado nos hace pensar que dio lugar a tensiones mecánicas importantes, similares a un hinchamiento en un lugar en el que el agua sólo puede llegar atravesando la galleta. Evidentemente, esa sustancia se ha formado allí; el artesano que fabricó el azulejo habría tenido buen cuidado en evitar tan destructora sustancia. No es fácil, por otra parte, comprender cómo una sustancia de tan deleznable aspecto pudo general los esfuerzos mecánicos suficientes para romper el vidriado; parece lógico suponer que actuaron sincronizados un efecto de despegado y un efecto de hinchamiento. Pasemos, pues, a estudiar el depósi-

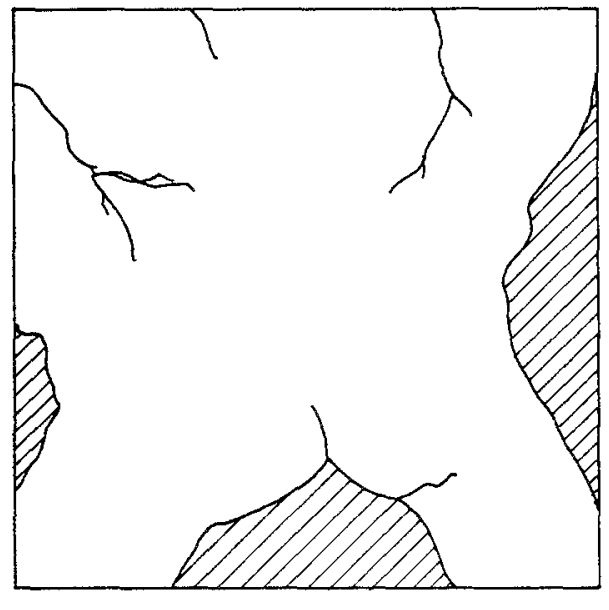

VISTA. FRONTAL

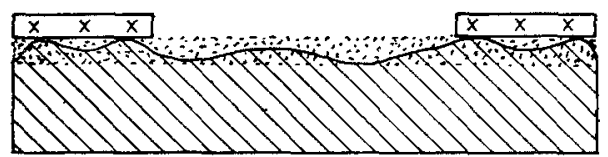

VISTA DE UN BORDE

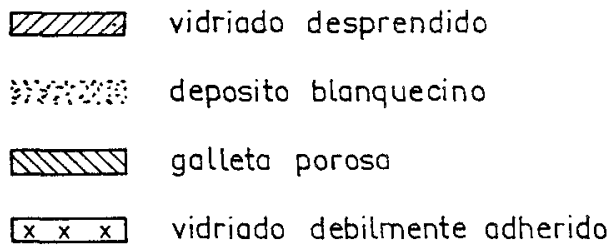

Fig. 1

to blanco que parece contener la clave del proceso deteriorador.

Pudimos obtener una reducida cantidad de muestra solamente. Por ello elegimos como técnicas analíticas la espectrometría de emisión y la difracción de rayos $\mathrm{X}$; no teníamos suficiente cantidad de muestra para realizar más análisis.

$\mathrm{El}$ análisis espectrométrico fue realizado con registro fotográfico (sometiendo al arco eléctico una diminuta pastilla de un compactado de polvo de grafito igual a los electrodos y una porción de la sustancia problema. El análisis es, pues, semicuantitativo, dejando excluídos elementos como oxígeno, hidrógeno, cloro, etc., como es habitual. La composición atómica resultó ser: 


\begin{tabular}{|c|c|}
\hline Magnesio & $0,2 \%$ \\
\hline Silicio ........ & $0,5 \%$ \\
\hline Molibdeno & No detectable \\
\hline Hierro .... & $0,3 \%$ \\
\hline Cobre... & $0,05 \%$ \\
\hline Manganeso, níquel, plata & No detectable \\
\hline 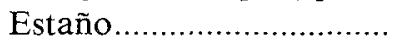 & $0,05 \%$ \\
\hline Plomo & $0,3 \%$ \\
\hline  & $1 \%$ \\
\hline $\begin{array}{l}\text { Vanadio, wolframio, cro- } \\
\text { mo y cinc....................... }\end{array}$ & No detectable \\
\hline 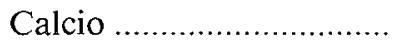 & $5 \%$ \\
\hline Sodio & Base (hasta $100 \%$ ) \\
\hline
\end{tabular}

El resultado indica que la sustancia no es arcillosa (bajo silicio, aluminio y magnesio), debiéndose de tratar de un compuesto de sodio muy impurificado, especialmente por calcio.

$\mathrm{El}$ análisis de difracción de rayos $\mathrm{X}$ se efectuó en cámara Debye-Scherrer, ya que la cantidad disponible de muestra no nos permitía hacer una pastilla compactada para el difractómetro. La intensidad de las líneas de difracción fue medida visualmente, por lo que los resultados numéricos del análisis deben entenderse como orientativos solamente. Las sustancias fueron identificadas confrontando el difractograma con las fichas estandard de la American Society for Testing of Materials.

$\mathrm{El}$ aspecto general de las rayas del difractograma indicaba una precaria perfección cristalina en no todos los compuestos que constituían la muestra (por ejemplo, la raya más intensa de todas correspondía a un amplio intervalo de espaciados atómicos desde 3,74 hasta $3,86 \AA$ ), con unos espaciados inicales de 7,73 $\AA$. Estos planos atómicos tan separados apuntan (en compuestos inorgánicos) hacia compuestos hidrolizados o hacia silicatos muy complejos.

Los resultados del análisis fueron:

\begin{tabular}{|c|c|c|c|}
\hline \multicolumn{2}{|c|}{ SUSTANCIA } & \multirow{2}{*}{$\begin{array}{c}\text { FICHA ASTM } \\
\text { UTILIZADA PARA } \\
\text { SU IDENTIFICACIÓN }\end{array}$} & \multirow{2}{*}{$\begin{array}{l}\text { CONTENIDO } \\
\text { EN \% } \\
\text { (APROX.) }\end{array}$} \\
\hline NOMBRE & FÓRMULA QUÍMICA & & \\
\hline Sulfato sódico & $\mathrm{Na}_{2} \mathrm{SO}_{4}$ & $3-0280$ & 53 \\
\hline Cloruro sódico & $\mathrm{NaCl}^{2}$ & $5-062$ & 28 \\
\hline $\begin{array}{l}\text { Sulfato sódico } \\
\text { decahidrolizado } \\
\text { Inidentificados }\end{array}$ & $\mathrm{Na}_{2} \mathrm{SO}_{4} \cdot 10 \mathrm{H}_{2} \mathrm{O}$ & $11-647$ & $\begin{array}{r}14 \\
5\end{array}$ \\
\hline
\end{tabular}

Estos resultados encajan perfectamente en el contexto del problema, tal y como lo habíamos planteado. La hidrolización del sulfato sódico, que tie-

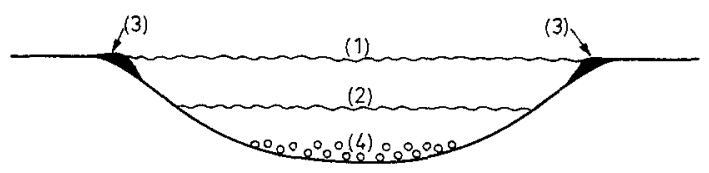

(1) Nivel del agua durante el lorgo periodo lluvioso.

(2) Nivel descendido, habiendo dejado en (3) su deposito seco de precipitados ultrofinos.

(4) Decontados gruesos.

a) Simit de un charco

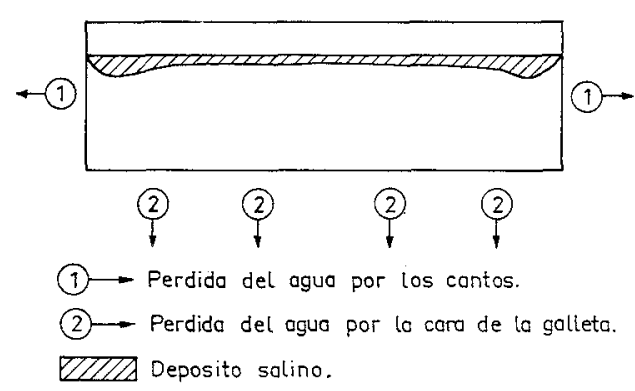

b) Deposito salino en el azulejo.

Fig. 2

ne lugar a temperatura ambiente sin ninguna dificultad, supone un incremento de volumen tan tremendo, que a nivel microscópico se convierte en un poderoso hinchamiento capaz de romper a flexión algo más robusto que el vidriado de un azulejo. En efecto, la sustancia blancuzca depositada tenía la clave del proceso del deterioro.

El paso siguiente es pensar con detalle cuál fue el mecanismo del deterioro, a fin de ponerle el mejor de los posibles remedios. De otro modo, se pueden cometer daños irreparables. Por ejemplo, a una persona poco experimentada se le podría ocurrir dar un prolongado lavado con agua a los azulejos (las tres sales son solubles), a fin de retirar los restos de sales; sería un error porque el «remedio» podría disparar el dañado del vidriado, si ya hay la suficiente cantidad de sulfato sódico acumulado en la zona de la galleta próxima al vidriado, lo cual es más que posible en un azulejo antiguo aparentemente sano. La captación de diez moléculas de agua por cada molécula de sulfato sódico da lugar a una tan grande multiplicación del volumen de los microcristales, que el efecto cuña entre el vidriado y la galleta sería imparable.

Parece más que razonable la explicación siguiente, que vamos a ilustrar en la fig. 2 con un simple modelo muy asequible, el depósito del finísimo lé- 
gamo en la orilla de un charco que se seca muy lentamente.

Imaginemos un charco que se forma después de una tormenta. El agua que se encharca queda totalmente enturbiada. Pasa el tiempo y el agua se va clarificando, y, como un perfecto separador que es (para materiales ligeros, claro, con polvos muy densos no funcionaría) va posando por orden de tamaños los corpúsculos antes suspendidos. Sólo quedan las partículas ultrafinas estables en suspensión que al tener que precipitar por la obligada evaporación producida por el calor ambiental, se acumulan en el nivel máximo que el agua alcanzó.

Algo parecido acontenció en los azulejos objeto de este estudio, con sus estaciones de atmósfera más y menos húmedas cíclicamente alternadas. La galleta porosa, si se la concede tiempo suficiente, tiende a impregnarse, en todo su volumen, de humedad hasta un nivel en consonancia con el ambiente. La entrada del vapor de agua tenía lugar por la cara libre de la galleta y por los cantos. El vapor condensaba por la noche (más fría) en el interior de los poros, disolviendo las sales dejadas hace años por la durísima agua con que el artesano amasó su arcilla y que estaba repartida uniformente por todo el volumen de la galleta.

Llegada la estación seca y calurosa, el azulejo sufría la correspondiente desecación. El agua abandonaba en estado de vapor la masa de la galleta por donde podía, que nunca era a través del impermeable vidriado. El depósito salino quedaba situado en la capa de galleta próxima al vidriado y más acentuadamente en el centro, marcando la cota máxima que la humedad alcanzó y que más tarde desecó. Igual que el secarse una mancha de humedad en el yeso de la pared, la frontera de la zona húmeda queda marcada por una cinta de suciedad característica.

Las subidas de humedad estacionales posteriores hicieron el resto. Ya no allegaban restos salinos de la masa de la galleta, pues había quedado lavada. Eran un aporte acuoso que parcialmente quedaba atrapado en forma de agua de hidrólisis del sulfato sódico. El engrosamiento en volumen del sufato sódico era incensante e irreversible. El efecto de cuña crecía inexorablemente. Mucho aguantaron los vidriados debido a que en el centro, donde más pronunciado era el empuje, tiene la membrana vítrea mayor posibilidad de estirado sin llegar a la rotura. Pero el resultado sólo podía ser el que ha sido.

Hoy en día tenemos una clara prueba. El vidriado separado no dejó una superficie de galleta firme

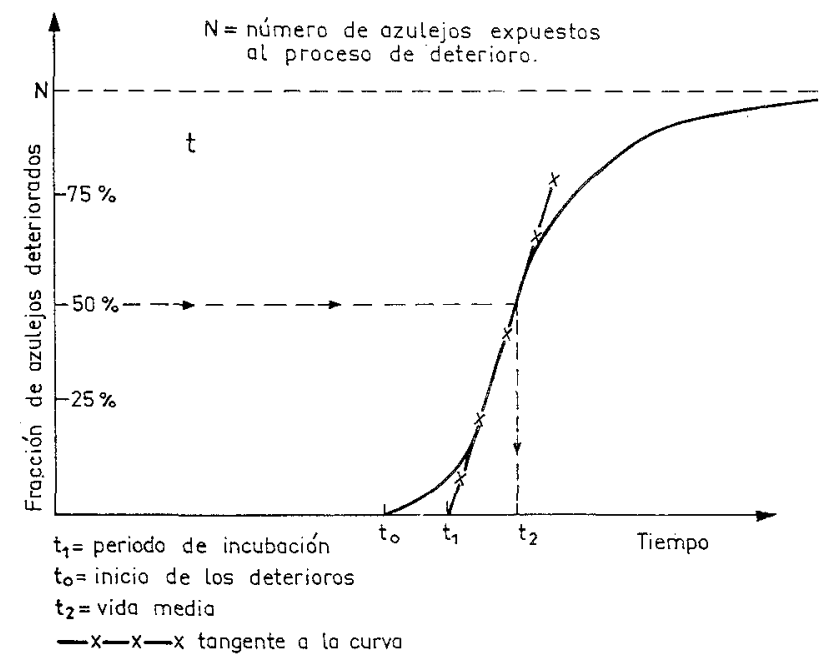

Fig. 3

y nueva. Dejó una cara de galleta pulverulenta, mucho más destrozada que la opuesta, libre de la «protección»del vidriado.

La pregunta que se nos viene a la mente es, si el mecanismo anterior es cierto, ¿cómo es que hay algunos azulejos que permanecen intactos? Hay varias respuestas, aunque lo más sensato es suponer lo siguiente:

De acuerdo con lo expuesto, el proceso de deterioro tiene dos etapas:

a) Período de acumulación progresiva y lenta del sulfato sódico en las proximidades del vidriado. Dura muchos decenios.

b) Período de hidrólisis del sulfato; se produce la rotura.

Es decir, estamos, lógicamente, ante un proceso de rotura que sigue una ley como se indica en fig. 3 , típica de fenómenos con proceso de iniciación actuando sobre poblaciones de probetas.

Es decir, aún en los aparentemente intactos, el proceso está en marcha; no han escapado a él. Para demostrarlo habría que romper un azulejo intacto y comprobar mediante análisis que el contenido en sales aumenta en la galleta a medida que nos aproximamos al vidriado. No merece la pena sacrificar una pieza para ello, pues el problema puede resolverse deshidrolizando las sales mediante un recocido. Veamos cómo.

Supongamos que a los azulejos a tratar les sometemos a un ciclo térmico tal y como indicamos en fig. 4.

La temperatura de recocido es tan baja que no afecta a la galleta ni al vidriado. Provoca sólo la 


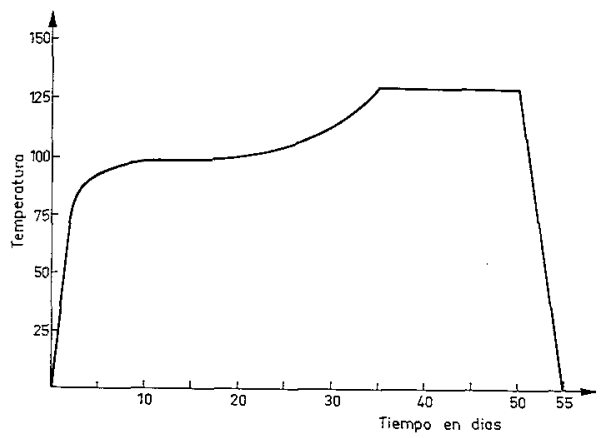

Fig. 4

reacción sulfato sódico decahidrolizado $\rightarrow$ sulfato sódico + agua, y permite que ésta tenga tiempo de escapar al exterior a través de la interminable red de túneles retorcidos que constituyen los poros de la galleta.

Al final del tratamiento tomaríamos a los aparentemente intactos, e impermeabilizaríamos a la superficie libre de galleta con un spray de cera, barniz tapaporos, laca transparente y mate, etc. Nunca más tendrá acceso al interior de la galleta la humedad ambiental del museo.

En cuanto a los afectados, limpiaremos con una minúscula espátula la superficie bajo el vidriado y accesible por rotura del mismo. Después podrán ser pegados en su sitio los trozos de vidriado sueltos (con una resina epoxi muy poco viscosa, y con curado a temperatura ambiente, por ejemplo). Después se impermeabiliza como en el caso anterior.

El tratamiento debe ser, lógicamente, dar marcha atrás en el proceso de dañado y poner el remedio para que no vuelva a producirse. Las cosas son muy sencillas, una vez explicadas, y se trata simplemente de saber servirse de las posibilidades que nos ofrecen la técnica y la ciencia de hoy día: no se trata de mal calificar a aquellos artesanos que produjeron aquellos tan atacables azulejos. Cualquier albañil de hoy día sabe que en el interior de una casa puede pegar los azulejos con yeso (un saco de yeso es sulfato cálcico en polvo, mientras que el yeso que cubre las paredes es sulfato cálcico hidratado. Tenemos, pues, la misma reacción con compuestos similares), pero en el exterior ha de ser con cemento (que reticula el agua en cristalización, por lo que la deshidratación del cemento no es posible). Si pegan azulejos con yeso en una pared exterior batida por la lluvia, al cabo de unos años aparecerán en los azulejos los mismos problemas de desvidriado que en los del museo.
Con esto no pretendemos decir que otros tipos de tratamientos (acondicionar en humedad y temperatura la atmósfera del museo, vitrinas rigurosamente herméticas y desecadas, etc.) sean ineficaces. Simplemente que con unos medios bien simples (una estufa con programación de temperatura existe en cualquier laboratorio) pueden remediarse unos problemas que han aparecido en este caso concreto, por sus especiales ciclos de temperatura-humedad-tiempo. Posiblemente, como no se presentan generalmente estos problemas, su tratamiento no está recogido en la literatura de museología.

\section{EL PROBLEMA DE LA DESTRUCCIÓN DE LOS CLAVOS QUE SUJETAN LAS CERÁMICAS COBREADAS}

En este museo existen unos platos de cerámica cobreada del siglo XVI expuestos en vitrinas para una cómoda observación por parte de los visitantes. La gran vistosidad de esta cerámica radica en su decoración con cobre brillante, pues en su fabricación se procedía a decorar con óxido de cobre, terminando la cochura con atmósfera reductora, de modo que se producía una reducción del cobre que quedaba depositado sobre el vidriado.

El problema de conservación en museo que estas magníficas cerámicas presentan es el siguiente: Estas piezas cerámicas permanecen en el interior de unas vitrinas de antiguo diseño (incómodas de desmontar y faltas por completo de estanqueidad) colgadas de la pared apoyándose en unas alcayatas o escarpias clavadas, que, por su forma impiden que la pieza se escurra hacia afuera y, al salirse del clavo, caiga al suelo de la vitrina y se quiebran tan valiosas piezas.

El problema que presenta este lógico y simple sistema de sujección es el siguiente: Al cabo de un cierto tiempo se observa una rapidísima destrucción de las alcayatas (son como las que habitualmente se venden en las ferreterías, de hierro) en la zona de contacto entre el cobre de las cerámicas y la alcayata.

Aparentemente, el cobre no sufre ataque o deterioro, pero el conservador se ve obligado a renovar de vez en cuando la escarpia, para lo que es obligado el desmontaje de la anticuada vitrina. Uno se pregunta si no existe alguna solución para el problema, que evite por completo el riesgo de caída y el trabajo del cambio periódico. 


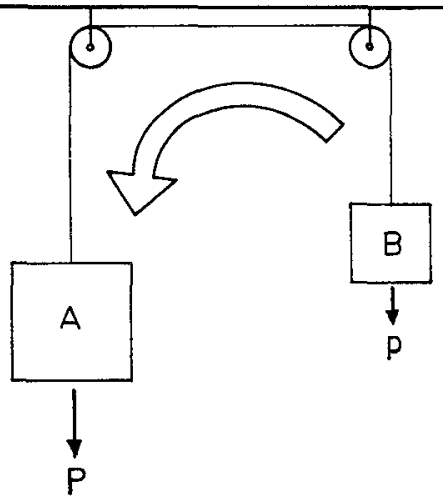

Fig. 5

Evidentemente, se trata de un problema típico de corrosión. Explicándolo de una forma muy elemental, cuando un metal origina la corrosión de otro de menor nobleza sucede como en el símil mecánico de la fig. 5 .

Las dos pesas tienden a caer, pero como están unidas por una cuerda, la pesa A arrastrará en su caída a la $\mathrm{B}$ (de menor peso) que tenderá a ascender, en contra de su tendencia natural a bajar hacia el suelo. Algo similar acontece con la oxidación de los metales.

Todos los metales están más estables en forma de óxido que como metal libre; en la naturaleza abundan los óxidos (el mineral más abundante con mucho es el cuarzo, que es óxido de silicio). Cuando dos metales se ponen en contacto, el más ávido de oxígeno se corroerá, mientras que el otro tenderá a aparecer como metal libre. Así, por ejemplo, los fundidores, cuando desean calentar una zona de un molde, colocan en ese punto una «termita», es decir, una mezcla de aluminio metálico y óxido de hierro bien mezclados. El calor del metal fundido inicia la reacción, mediante la cual el aluminio se oxida y el hierro se libera del oxígeno. La reacción es exotérmica y aporta un calor importante que ayuda a mantener el metal en estado líquido en una zona en la que la solidificación temprana del metal hubiese originado un atasco en el flujo del caldo.

Así, pues, la avidez por el oxígeno juega en estos procesos el papel de peso de las pesas de nuestro símil anterior. La afinidad de los metales por el oxígeno es de sobra conocida, pues no es más que la tan divulgada serie electroquímica, que exponemos a continuación:

Oro
Platino
Plata
Mercurio
Cobre
Bismuto
Antimonio
Plomo
Estaño
Níquel
Cobalto
Hierro
Cadmio
Cromo
Cinc
Manganeso
Aluminio
Magnesio
Sodio
Potasio

Grado de nobleza

(Propensión a liberarse del oxigeno)

Ahora comprendemos porqué a los cascos de los actuales barcos de hierro se les adhieren «ánodos de sacrificio» de cinc postizos que reponen de tiempo en tiempo. Mientras exista cinc en proceso de corrosión, la totalidad del hierro del casco se comportará como «noble», es decir, no se corroerá. La cómoda reposición períodica de los ánodos de sacrificio nos salva a la totalidad del casco del buque de la agresión del agua del mar.

Mirando ahora en la tabla la posición relativa del cobre y del hierro comprenderemos que en las vitrinas de nuestro museo, debido a la humeada ambiental, los clavos están actuando de ánodos de sacrificio frente al cobre de las piezas cerámicas. Por ello no se aprecia dañado alguno en el cobre de los vidriados.

Veamos la forma de solucionar este problema de corrosión. Las posibles vías de actuación son:

a) Eliminar la humedad ambiental, pues a temperatura ambiente (a altas temperaturas he- 
mos visto el ejemplo de la termita) dos metales secos no interaccionan. Ello obligaría a unas vitrinas rigurosísimamente estancas y a una desecación interna exhaustiva. Es una solución válida, aunque enojosa. Más vale propiciar el que las piezas se encuentren en equilibrio bajo el ambiente normal del museo, aunque éste no sea el óptimo deseable.

b) Poner unas alcayatas plateadas (recubiertas de plata). Quedaría resuelto el problema de la destrucción progresiva y peligrosa de las alcayatas, con el creciente peligro de caída y rotura de las piezas. Pero sería una solución nefasta, pues inmediatamente comenzaría el ataque del cobre del vidriado de las piezas cerámicas.

c) Dar un recubrimiento a la alcayata de los habituales en hierro expuesto a la intemperie (galvanizado con cinc, cromado, niquelado, etc.). Dada la nobleza relativa del cobre, estos recubrimientos, que normalmente resisten la corrosión atmosférica, resultarían dañados. Después continuaría la corrosión del hierro de la alcayata.

d) Recubrimiento con cobre. Posiblemente sea una solución perfecta. Recubriendo a la alcayata con cobre se evita la posibilidad de cualquier interacción escarpia-vidriado. El recubrimiento puede hacerse, entre otros procesos posibles, por:

i) inmersión en un líquido de composición.

Sulfato de cobre .............. $35 \mathrm{~g} /$ litro

Sal de Rochelle ................. 150 g/litro

Hidróxido sódico .............. 80 g/litro

ii) electrodeposición con un baño de tipo. Cianuro de cobre $30 \mathrm{~g} /$ litro
Cianuro sódico

$37,5 \mathrm{~g} /$ litro

Sal de Rochelle

$37,5 \mathrm{~g} /$ litro

Carbonato sódico

$35 \mathrm{~g} /$ litro

Temperatura

50 a $70^{\circ} \mathrm{C}$

Densidad de corriente.

20 a 60

$\mathrm{mA} / \mathrm{cm}^{2}$

Este segundo proceso es más aconsejable que el primero porque permite proteger con un recubrimeinto de cobre más grueso que con el proceso primero.

e) Recubriendo a la alcayata con una resina eléctricamente aislante, dura e impermeable. Bastará adquirir un adhesivo, por ejemplo, tipo epoxi en dos componentes (en el comercio son populares los dos tubos de marca Araldite, Nural, etc., cuyo contenido se mezcla a partes iguales antes de ser usado) con el cual se recubre a modo de pintura a las alcayatas ya clavadas. Cuarenta y ocho horas más tarde pueden colocarse los platos en las alcayatas y el problema quedará satisfactoriamente resuelto.

Evidentemente, las soluciones d) y e) son perfectamente practicables. Una vez más, si nơs hubiéramos enfrentado al problema sin ideas claras, podríamos haber caído en una solución tal y como la c), bastante deficiente. Hay siempre en estos casos soluciones muy eficaces y fáciles de realizar.

\section{AGRADECIMIENTO}

Agradecemos a la Dirección del Museo Provincial de Murcia el permiso para publicar el presente trabajo. 